POS $\quad \begin{aligned} & \text { PROCEEDINGS } \\ & \text { OF SCIENCE }\end{aligned}$

\title{
A Proposed Evolution of the Mu2e Experiment
}

\section{Craig Group*†}

University of Virginia

E-mail: rcg6p@virginia.edu

The 20th International Workshop on Neutrinos (NuFact2018)

12-18 August 2018

Blacksburg, Virginia

* Speaker.

${ }^{\dagger}$ On behalf of the Mu2e collaboration. 
Searches for rare processes and precision measurement have become an important component of the particle physics research program hosted on US soil. These efforts share the need for intense beams (the Intensity Frontier [1]). The Mu2e experiment, to be hosted at Fermilab, will search for the charged-lepton-flavor-violating (CLFV) process of coherent muon-to-electron conversion in the presence of a nucleus with a sensitivity four orders of magnitude beyond current limits [2]. In this talk, we first motivate the search for charged-lepton-flavor violation, then describe the firstgeneration plan for the Mu2e experiment, and finally mention some of the upgrades required for a second generation aimed at improving the sensitivity by a factor of ten.

\section{Charged Lepton Flavour Violation}

In a sense, the muon has been a thorn in the side of particle physicists since its discovery in 1936. We are still seeking answers related to questions of flavor: Why three families? Why are the lepton masses so different? Are there other differences between flavors? Experiments searching for CLFV seemed to run out of huge sensitivity gains in the 1990s. However, a rebirth is ongoing! A new series of experiments, led by new technologies, are poised to push sensitivities down several orders of magnitude. Soon, the muon sector may lead to discoveries that will help answer the most fundamental questions of particle physics.

Experiments using muons to search for CLFV have been constructed to search for $\mu \rightarrow e \gamma$, $\mu^{+} \rightarrow e^{+} e^{-} e^{+}$, and the coherent $\mu^{-} N \rightarrow e^{-} N$ conversion process in nuclei. The present state of lepton-flavor violation searches using muon decay is: $\operatorname{Br}\left(\mu^{+} \rightarrow e \gamma\right)<4.2 \times 10^{-13}[3], \operatorname{Br}\left(\mu^{+} \rightarrow\right.$ $\left.e^{+} e^{-} e^{+}\right)<1 \times 10^{-12}[4], R_{\mu e}<7 \times 10^{-13}[5]^{1}$. There is potential to improve each of these searches significantly over the next decade.

Many SM extensions $[6,7,8]$ include CLFV interactions that would force this process to occur at a rate much higher than the one expected in the SM. In fact, many new physics models predict a conversion rate that might be observed experimentally at Mu2e.

Searches for $\mu^{-} N \rightarrow e^{-} N$ and $\mu \rightarrow e \gamma$ are complementary. If a signal is observed, then the relative rates of $\mu \rightarrow e \gamma, \mu^{+} \rightarrow e^{+} e^{-} e^{+}$, and the coherent $\mu^{-} N \rightarrow e^{-} N$ conversion processes can be used to constrain the interactions that are responsible for the signal [9]. Searches for CLFV are also complimentary to LHC searches. In many discovery scenarios at the LHC, it is difficult to determine if lepton flavor is violated. However, in an LHC discovery scenario with $R_{\mu e} \approx 10^{-15}$, Mu2e would observe about 40 events on a background of fewer than one event. In addition, if the experiments at the LHC do not find a signal of new physics, Mu2e will probe energy scales up to thousands of $\mathrm{TeV}$, so, it may be our best chance to probe physics beyond the standard model.

\section{The Mu2e Experiment}

The Muon-to-Electron-Conversion (Mu2e) Experiment is a high-precision, intensity-frontier experiment being developed at Fermilab that will search for coherent, neutrino-less muon to electron conversion in the presence of an atomic nucleus. Mu2e will improve the sensitivity by four

\footnotetext{
${ }^{1} \mu^{-} N \rightarrow e^{-} N$ searches set a limit of $R_{\mu e}$. The ratio of $R_{\mu e}$ is defined by, $R_{\mu e}=\frac{\Gamma\left(\mu^{-} N(A, Z) \rightarrow e^{-} N(A, Z)\right.}{\Gamma\left(\mu^{-} N(A, Z) \rightarrow v_{\mu} N(A, Z-1)\right)}$, where $N(A, Z)$ denotes a nucleus with mass number $A$ and atomic number $Z$. The numerator is the rate for the conversion process and the denominator is the rate for ordinary muon capture on the same nucleus.
} 
orders of magnitude over the present limits with planned sensitivity goal of approximately $10^{-16}$. In the search for beyond the standard model (BSM) physics, Mu2e is uniquely sensitive to a wide range of models by indirectly probing mass scales up to the energy scale of $10^{4} \mathrm{TeV}$. While muonto-electron-conversion is permissible through neutrino oscillations, the rate is unobservable with an expected rate of only about one event in $10^{52}$. By design, the background for the experiment will be well-understood and kept at a sub-event level, which means that the observation of muonto-electron conversion would be an indirect discovery of BSM physics.

\section{A Proposed Upgrade to the Mu2e Experiment}

An evolution of the Mu2e experiment has been proposed, called Mu2e-II [10, 11]. The experiment will leverage advances in detector technology and use the increased proton intensity provided by the Fermilab PIP-II upgrade [12] to improve the sensitivity by one order of magnitude beyond the Mu2e experiment, providing the deepest probe of charged lepton flavor violation in the foreseeable future. Mu2e-II will use as much of the Mu2e infrastructure as possible, only making improvements to the Mu2e apparatus to accommodate the increased beam intensity and cope with the accompanying increase in backgrounds.

An upgrade to Mu2e by a factor of ten in sensitivity is well founded regardless of the physics result from Mu2e. If Mu2e sees a strong signal, measuring the rate versus the $\mathrm{Z}$ of the target material can be used to help sort out the nature of the discovery. If a weak signal is observed, then the statistical accuracy of the result can be improved with more data. Finally, if no signal is observed at Mu2e, extending the sensitivity could reach a signal or set even stronger limits.

Mu2e-II proposes to run as at lower beam energy $(800 \mathrm{MeV})$ relative to $\mathrm{Mu} 2 \mathrm{e}(8 \mathrm{GeV})$ and use a $\mathrm{H}$ - beam. By improving the duty factor from $25 \%$ to approximately $90 \%$ Mu2e can increase the number of stopped muons by a factor of 10 while only increasing instantaneous detector rates by about three times the nominal Mu2e instantaneous rates. Suppression of out-of-time protons must also be improved by a factor of ten over Mu2e.

Several potentially costly challenges exist and require $R \& D$ to ensure systems that are capable of enabling the Mu2e-II physics goals. A new beam line will be required to transport proton beam from PIP-II to the Mu2e production target station, and beam optics are more difficult for the lower energy beam. A radiatively-cooled target, as planned for Mu2e, will not be adequate for the increased beam intensity. The heat and radiation shield of the muon production solenoid for Mu2e must be upgrade/replaced for Mu2e-II. Detector front-end electronics may be challenged by radiation damage. The momentum resolution must be improved in order to keep backgrounds to Mu2e levels. To improve resolution, thinner stopping target foils and the feasibility of thinner straw walls in the tracker should be considered.

In summary, the proposed Mu2e upgrade is well matched to take advantage of the Fermilab PIP-II accelerator upgrade and the experimental infrastructure of Mu2e. With required upgrades, Mu2e-II will be positioned to either probe discoveries or improve the search reach of Mu2e. In either case Mu2e-II is well motivated and is a natural step in the search for CLFV.

\section{References}

[1] J. L. Hewett et al., arXiv:1205.2671. 
[2] L. Bartoszek et al. [Mu2e Collaboration], arXiv:1501.05241.

[3] A. M. Baldini et al. [MEG Collaboration], Eur. Phys. J. C 76, no. 8, 434 (2016).

[4] U. Bellgardt et al. [SINDRUM Collaboration], Nucl. Phys. B 299, 1 (1988).

[5] W. H. Bertl et al. [SINDRUM II Collaboration], Eur. Phys. J. C 47, 337 (2006).

[6] W. J. Marciano, T. Mori and J. M. Roney, Ann. Rev. Nucl. Part. Sci. 58, 315 (2008).

[7] Y. Kuno and Y. Okada, Rev. Mod. Phys. 73, 151 (2001).

[8] L. Calibbi, A. Faccia, A. Masiero and S. K. Vempati, Phys. Rev. D 74, 116002 (2006).

[9] A. de Gouvea and P. Vogel, Prog. Part. Nucl. Phys. 71, 75 (2013).

[10] K. Knoepfel et al. [mu2e Collaboration], arXiv:1307.1168.

[11] F. Abusalma et al. [Mu2e Collaboration], arXiv:1802.02599.

[12] PIP-II CDR, Appendix A (http://pxie.fnal.gov/PIP-II_CDR/default.htm). 